\title{
8 \\ The Experience of Left and Right
}

\author{
Geoffrey Lee
}

A transcendental argument is an argument of the form 'Experience has feature $\mathrm{F}$. If experience has feature $F$, then the external world has feature G. So the external world has feature G.' Kant and others have attempted to use arguments of this form to argue against sceptics about the existence of the external world. ${ }^{1}$ In this chapter I'm not going to argue against any sceptics, but I'm going to show you how certain very plausible assumptions about experience can lead to surprising conclusions about how certain experiences are physically realized. In particular, you'll see how to travel transcendentally from facts about experience to facts about the spatial properties of the brain. ${ }^{2}$

But to get that far will take us a while. To begin with, let me remind you of the frequently discussed possibility of 'spectrum inversion' ${ }^{3}$ The familiar hypothesis is that the qualitative character of your colour experience might differ systematically from mine. So, for example, when I see a red object, my colour experience might be qualitatively like yours is when you see a green object, and vice versa. Further, it is claimed, since there is a (non-trivial) isomorphism from the colour-space on to itself that preserves all the basic qualitative similarities and differences between colours, this could occur without resultant differences in our (ordinary) behaviour. It is this supposed symmetry that is meant to allow for the possibility of non-manifest spectrum inversion.

The starting point for my discussion is the idea of qualitative inversion with respect to another perceived feature of the local environment-its left/right

I would like to thank the following people for helpful and inspiring comments and discussion: Ned Block, David Chalmers, Shamik Dasgupta, Tamar Szabó Gendler, John Hawthorne, Rory Madden, Chris Peacocke, Derek Parfit, Adam Pautz, Richard Price, Carlos Schafer, Sydney Shoemaker, Declan Smithies, Sebastian Watzl, and two anonymous referees (you know who you are).

1 Or, our knowledge thereof. Transcendental arguments typically have conclusions like 'there is an external world', or 'I am not a brain in a vat', rather than, e.g., 'I know that there is an external world'.

2 Martin Davies (1992) has attempted to run a similar kind of argument, in his case from armchair considerations to the conclusion that there is a Language of Thought.

3 For a classic discussion, see Shoemaker (1982). 
orientation about the viewer. ${ }^{4}$ The idea is that there could be intra- or intersubjective inversion with respect to the 'reflective orientation of the world', so that the way things appear to you when actually on the right is the way they appear to me when actually on the left, and vice versa. ${ }^{5}$ It should be less controversial that there is a symmetrical structure to the qualitative space of left-right experience. This symmetry can be exploited to generate a different kind of experiential inversion hypothesis.

So, for example, when you look in a mirror, the apparent reflective orientation of the world gets inverted with respect to vision. Writing appears backwards, a chair that is on the left-hand side of the room appears on the right-hand side, and so on. Of course it's normally quite easy to tell that you are looking in a mirror. For example, one important cue is that items apparently in the mirror world appear to change position systematically with a change in position of the subject, in a way that would not occur if they were instead being viewed through a transparent pane of glass.

But although such cues exist when this inverting effect is produced by mirrors, perhaps experience could be left/right inverted in such a way that no such cues exist. (In order for this to occur without behaviourally manifest consequences, all the senses would need to be suitably inverted, not just vision. $\left.{ }^{6}\right)$

So, to be explicit, when I say things like 'the possibility of reflective inversion' in this chapter, I mean the following:

The Inversion Hypothesis: There could be a subject exactly like me in his classificatory and discriminatory dispositions, and in his ability to navigate the world, but who is such that, when under normal conditions he is presented with a particular external stimulus, he has experiences that are left/right inverted with respect to those that I would have under normal conditions, when presented with the same stimulus.

I will call the experiences of someone who is thus inverted with respect to me '(non-manifest) mirrored experiences' and I will refer to such a person as being '(non-manifestly) mirror-inverted'. (I will omit the parentheticals unless context requires their inclusion.)

The point of considering this hypothesis rather than the usual colour inversion hypothesis is that we will be led to certain surprising results already alluded to, results that do not have obvious analogues in the colour case. So what I am

${ }^{4}$ After completing this paper, I discovered that Susan Hurley has a related discussion of left/ right inversion in her (1998) - see, in particular, ch. 8.

5 I should note the related idea of up/down inversion, although I think it's much harder to make sense of non-manifest up/down inversion than left/right inversion. Interestingly, on at least one way of spelling out what up/down inversion would amount to, it is just the same thing as left/right inversion.

${ }^{6}$ Note that there also has to be an inversion of the causal relationships between (narrowly construed) intentions to move and bodily action if the experientially inverted subject is to successfully navigate the space around her. 
interested in is not just the truth of Inversion Hypothesis (although I will present some arguments for it), but also certain other results about left/right experience that will be uncovered as we proceed. Moreover, the case of left/right inversion can be used to make most of the same points that the inverted spectrum example is frequently used to make, but less controversially. It also has the interesting feature that left and right are spatial relations and not sensory qualities-this means that certain ways of characterizing the inversion phenomenon that appeal to the specifically sensory nature of colours will not be available in this case.

But before entering the main discussion, I need to show that non-manifest mirrored experiences are at least prima facie conceivable (they are definitely quite hard to imagine).

\section{THE CONCEIVABILITY OF MIRRORED EXPERIENCES}

I have found that when some people consider the hypothesis of mirror inversion, they doubt whether there could be a coherent train of mirrored experiences. Perhaps the world could look inverted if I were just gazing in one direction, but could inverted experiences be consistently produced if I started to move around? Let me explain what these experiences would be like with the following example (the example will also serve as a basis for later considerations).

Take the layout of objects, floors, ceilings and walls in my apartment. It is obviously possible that an exact mirror image of my apartment could be constructed, and mirror-image versions of all the objects inside be put in appropriate counterpart locations. Furthermore, it seems obvious that if you were to wander into this mirror apartment, things would look very different from the way they would look if you wandered into my actual apartment.

Note that this mirror apartment would look like a reflection of my apartment viewed from any counterpart position within the apartment at any counterpart angle. It is an elementary result of geometry that the mirror image of a figure that results from reflecting it through any one chosen plane can be mapped on to the mirror image that results from choosing any other plane as the reflecting plane, just using rotation and linear movement operations. Thus whatever plane we chose to reflect the apartment through, the mirror apartment we would construct as a result would be intrinsically the same.

We can use this set-up to describe the experience of an individual who is mirror inverted. ${ }^{7}$ The character of his experience as he walks through the door into my actual apartment would be the same as the character of your experience as you walk through the door into the mirror apartment. As he moves along any

\footnotetext{
7 Likewise, one might explain the colour inverted spectrum hypothesis by saying that your experiences might be just like the ones that I would have if I were in an environment exactly like this except that all the colours of the external objects had been systematically switched.
} 
path through my apartment, his experience is the same as yours would be if you traced the mirror path around the mirror apartment. And, given the simple geometrical point made in the previous paragraph, we can see that at every stage in this path, his experience would be a 'mirror' of the experience you would be having in your counterpart journey.

This description of an inverted subject's experience is not restricted to vision. For example, the qualitative character of the overall experience he would have if he stood in the middle of the apartment and turned around clockwise in a complete circle would match the one you would have if you stood in the middle of the mirror apartment and turned anticlockwise in a complete circle. Here it's not just the visual experience that would feel the same, but also the proprioceptive experience and action awareness associated with turning the body. Or, to take another example, if someone were to throw a ball at him that hit his left elbow, he would have an experience qualitatively the same as the one you would have if a similarly thrown ball were to hit your right elbow. Likewise with sounds, smells, tastes, etc. A triumphant peal of bells coming from the left would sound to this subject the way it would to you coming from the right; a bitter crystal dissolving on the left side of the tongue would feel like it would to you dissolving on the right side, and so on.

I will now consider some interesting arguments designed to show how mirrored experiences might be physically realized. These will serve to show that, on certain assumptions, the Inversion Hypothesis is true.

\section{MIRROR MEN}

Consider first the following puzzle. Take the brain of a subject who is in the midst of a train of conscious experiences. Suppose we also had another subject's brain that was exactly like the brain of this subject, except that the matter was arranged in a mirror image. ${ }^{8}$ The question is: could there be any difference between the experiences being had by the two subjects? A closely related question is the following: suppose it were possible to physically reflect your brain in the middle of a course of experiences, but hold all the other physical facts about the brain constant. Would there be any difference between the experiences that ensue and the ones you would have had if your brain hadn't been flipped? (We should avoid being seduced here by a misguided picture of experience as involving a super-rigid homunculus gazing at an intra-skullular cinema screen that would be inverted by the flipping.)

The answers we can give to these questions are constrained by the view we take of the metaphysics of left and right. Let me briefly sketch the main views.

8 There are certain difficulties that arise with this idea to do with the possibility of laws of nature that are sensitive to the left/right distinction. They will be dealt with later. 
Consider a left glove. There are two basic views about what makes it lefthanded. According to one view, call it 'absolutism', what makes a glove left or right is its possession of an intrinsic shape property of 'right glovedness' or 'left glovedness'. On this view there is an intrinsic difference between a left glove and a right glove, and every glove has one of these two reflective properties.

The other view, call it 'relationism', is rather more subtle. On this view, there is no intrinsic non-relational difference between a left glove and a right glove. All there really is, is the relation of congruence-that is, the relation that two gloves stand in when they are of the same glovedness. There are no intrinsic properties such as 'left glovedness' and 'right glovedness', so a left glove and right glove are intrinsically the same.

Of course, the absolutist and relationist both agree that the congruence relation exists; what is affirmed by the absolutist and denied by the relationist is that when two gloves stand in that relation their so doing is grounded by a difference in intrinsic properties. According to absolutism every glove has an intrinsic reflective property, either 'left glovedness' or 'right glovedness', and relations of congruence are supervenient on the distribution of these intrinsic properties. According to relationism there are only reflective relations between gloves, which are not grounded on the objects having any intrinsic reflective properties.

A closely related distinction is between those who think there are inter-world facts about congruence, and those who do not. For example, Kant mentions a world containing a single lonely hand. ${ }^{9}$ Is there any fact of the matter about whether it is a left hand or a right hand? If you think that facts about inter-world sameness and difference depend only on the distribution of properties and relations within worlds, then you will think that there could only be such a fact of the matter if absolutism were true. Similarly, a relationist is likely to hold that there is no real difference between any two worlds that purportedly differ only in that one is arranged as a mirror image of the other. Strictly speaking, there is no contradiction in a relationist not saying this, but that would commit him to the view that there are brute modal facts that do not supervene on the distribution of non-modal facts.

Whether you are an absolutist or a relationist makes a difference to how you answer my questions about the experiences of incongruently brained subjects. For if you are a relationist, then you hold the position that two incongruous counterpart brains are intrinsically identical. If you also suppose that the character of experience supervenes on the intrinsic character of the brain, then you are forced into the position that the experiences being had by the owners of the different brains are the same. If you are an absolutist, then you think there is an intrinsic difference between the brains. So the same supervenience claim does not commit you to saying that the experiences in the two subjects are the same. The

9 See Kant (1991) for his classic 1768 discussion of this point. 
answer you give to these questions then depends on whether you believe that intrinsic reflective properties could be part of the minimal base of physical properties that determine the experiences being had by the owner of the brain.

The same kinds of questions arise even if you considerably weaken the supervenience claim, as some philosophers with externalist tastes are wont to do. Suppose, for example, that you only claim that the qualitative character of experience supervenes on the intrinsic character of the whole subject and his local environment (so, in particular, this intrinsic character includes relations between the subject and the objects that he is experiencing). You can then compare this set-up with an incongruous counterpart of the set-up, involving the subject and his environment laid out in a mirror image. Again, if you are a relationist, you will be committed to the view that the experiences being had in the two places are the same.

\section{THE RELATIONIST ARGUMENT}

Keeping these points in mind, I will now present a more complex argument that bears more directly to the question of whether inter-subjective reflective inversion of experiences is possible. I'll call this the 'Relationist Argument'. I'll call it that because it's supposed to show that if relationism is true then the Inversion Hypothesis is true. A bit later on we will consider what happens if we suppose absolutism to be true.

So let us take relationism as a premise. The Relationist Argument again considers the difference between the experiences of a subject and a mirror-image copy of this subject, although this time it's the whole physical subject we will be considering, not just a brain. Another difference is that we will only assume that the subjects are incongruous immediately prior to the stimulation that causes the experiences that we will compare. To anticipate, the conclusion of the argument is that a subject who was initially a physical reflection of you (your mirror twin) would, when presented with the same stimulus as you, have an experience that was inverted with respect to yours.

The gist of the Relationist Argument is as follows. If we were to reflectively invert the whole world around you the subject, then it seems like that would make a big difference to its appearance. For example, if you were about to read a sign that said 'MIT', then if we reflected the whole world around you, the sign would instead read 'TIM'. But if we are relationists, then there is no real difference between reflecting the world around you, and reflecting you the subject, holding the world constant. So we can conclude that if we made a mirror copy of you, he or she would have experiences like those you would have if the world around you were flipped over.

The Relationist Argument can be illustrated with a 'toy model' of how experience might depend on the physical realm. Imagine a group of subjects 
whose heads are painted red on the left side and white on the right side. These striped subjects are able to have experiences just like ours. But what happens when such a subject experiences an environment is that an image of the scene in front of them is literally imprinted on their forehead. So if a bed is on the subject's left, and a kite on his right, then the bed will imprint on their red side, and the kite on their white side. And the experiences the subject has of any scene obey the following rule: which experiences the subject has supervenes on the layout of the imprinted image with respect to the red and white portions of their head.

So suppose we have one of these red and white beasts, and let us christen him 'Striped Lefty'. Compare the imprinted pattern that Striped Lefty would get of this 'bed and kite' stimulus with the pattern that would result from him being exposed to the incongruent counterpart of the stimulus. Had the environment been arranged mirrorwise, Striped Lefty would have instead ended up with a kite image imprinted on his red side and a bed image imprinted on his white side.

Note that due to the asymmetrical distribution of colour on Striped Lefty's head, this second pattern on his forehead is neither identical to, nor the incongruous counterpart of, the pattern caused by the first stimulus; this time the bed image is white and the kite image is red. Indeed, that is just what we require if his experience of the mirrored stimulus is to be different from that of the original stimulus and we are assuming relationism.

Now suppose we build a mirror-image counterpart of Striped Lefty, as he was prior to being imprinted. Let us christen this new beast 'Striped Righty'. Striped Righty starts off life with the right side of his head red, and the left side of his head white. If we expose him to the 'bed on left, kite on right' stimulus, then he will end up with a bed image imprinted on his white side, and a kite image imprinted on his red side. In other words, he will end up in a state that is physically a mirror image of the state Striped Lefty was in when we exposed him to the opposite 'kite on left, bed on right' stimulus. But if we are relationists, this is exactly the same physical state. So it follows that Striped Righty's experience of the 'bed on left, kite on right' stimulus is the same as Striped Lefty's experience of the 'kite on left, bed on right' stimulus. And the same is true of any other stimulus and its incongruent counterpart. In other words, Striped Righty is experientially mirror inverted with respect to Striped Lefty.

Note that the physical state Striped Righty goes into when presented with any stimulus is neither identical to, nor the incongruous counterpart of, the state Striped Lefty goes into when presented with the same stimulus. So there is no contradiction here in holding that relationism is true and that Striped Righty and Striped Lefty would have mirror-inverted experiences of the same stimulus. (We would be in trouble, however, if both sides of Striped Lefty's head were the same colour to begin with; more on that shortly.)

The argument seems to work just as well if we consider instead the actual supervenience base for experience rather than the red/white image imprinting model. So suppose this time that we have just a normal human being, who we'll 
christen 'Simple Lefty' (or 'Lefty' for short). Suppose at t1, Lefty has his eyes closed ${ }^{10}$ and is about to view my apartment, and that at $\mathrm{t} 2$ the apartment viewing begins. Let 'Simple Righty' ('Righty' for short) be a mirror image copy of Lefty at $\mathrm{t} 1$.

The following statement is surely true:

(A) Suppose at t2 Lefty is viewing my apartment as it actually is. Then it is true that if the apartment had been laid out in a mirror image of the way it is actually laid out, his experience of it would have been reflectively inverted with respect to the experience he is actually having of it.

Now suppose that we are relationists about left and right. We might think that the following two hypotheses are really the same, in so far as the physical layout of the respective universes is concerned. ${ }^{11}$

(1) At t2, Lefty has just opened his eyes, and is standing gazing at the reflected version of my apartment, with the whole physical universe around the apartment also reflected.

(2) At t2, Righty has just opened his eyes, and is standing gazing at my apartment, as it actually is, from the perspective equivalent to the one Lefty is gazing from in scenario (1).

Add in the third scenario:

(3) At t2, Lefty has just opened his eyes, and is standing gazing at my apartment, as it actually is, from the same perspective as in (2).

Add in a fairly weak supervenience claim:

(WS): The qualitative character of a subject's experiences supervenes on the layout of physical properties of the whole universe.

We can now argue that the Inversion Hypothesis is true. It follows from (A), (WS) and the physical sameness of (1) and (2) that the experiences had in (2) and (3) are mirror-inverted with respect to each other. But they are experiences of exactly the same environment from the same perspective (and we can stipulate that conditions are quite normal). So we seem to have established at least one possible case of inter-subjective reflective inversion. Therefore, if relationism is true, then the Inversion Hypothesis is true.

The argument gives a physical sufficient condition for a mirrorwise difference in experiences - an individual who is initially a physical mirror duplicate of another individual will have experiences that are left/right inverted with respect to that individual. This is a strict sufficient condition. If we found individuals whose brain structure appeared to be built roughly in a mirror image to that

${ }_{10}$ Or, better, he is in a state of total sensory deprivation.

11 Here we can ignore the possibility of haecceitistic differences between objects. 


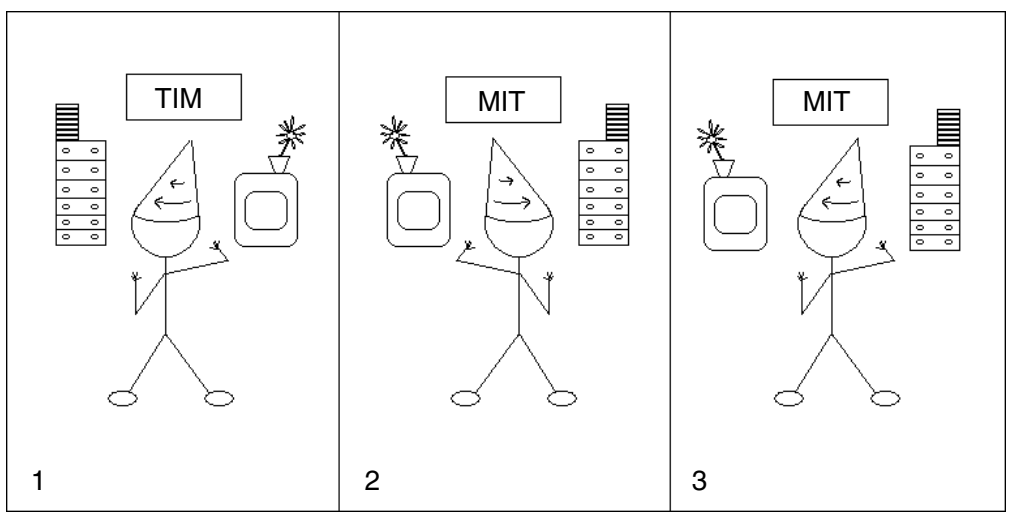

Fig. 1. Three hypotheses.

found in normal humans, that might be thought by a proponent of the Relationist Argument to be empirical evidence that they were experientially left/right inverted. (And indeed I am told that this is the situation with certain left-handed people.)

As I set it up, the Relationist Argument assumes relationism. In fact it is obvious that we could weaken this assumption to: 'if there are absolute reflective facts, then they are irrelevant in the determination of experience', and the argument would be equally good. So, if you're an absolutist, you can still consistently use the Relationist Argument, as long as you don't think absolute reflective facts are part of what determines experience.

\section{INVERSION AND FUNCTIONAL EQUIVALENCE}

An objection to the Relationist Argument which I should mention straight away is as follows: the complaint is that this could not be a genuine case of 'inverted qualia', because at the time when they are having inverted experiences of the same stimulus, Lefty and Righty are in physically quite different states (they are no longer merely incongruent), and so might be functionally quite different. But aren't inverted qualia cases supposed to be examples of functionally identical subjects who nevertheless have different qualia? ${ }^{12}$

The issues here are similar to those that arise in the case of colour, so I will be brief. My reply to this point is that we must distinguish different levels at which two subjects might be functionally alike. The conventional inverted spectrum scenario is normally only presented with a commitment to the subjects being functionally the same at the common-sense level of classification, discrimination, navigation and so forth. Such functional sameness is compatible with the

${ }^{12}$ Ned Block, David Chalmers, and an anonymous referee all stressed this point to me. 
subjects being functionally distinct at some deeper level on which the difference in qualia depends. So, for example, Shoemaker advertises himself as a proponent of the possibility of inverted qualia, even though he is a physicalist, and a functionalist about all physical properties.

Now I would claim that if the Relationist Argument goes through, Lefty and Righty are relevantly functionally alike, so should count as an example of inverted qualia. Righty's basic sensorimotor capacities are the same as Lefty's; for example, it is not the case that if Righty attempts to reach for an object on the left, he will reach to the right - he will succeed in basic navigation just as well as Lefty. Having said this, I should admit that there may be some functional differences at a fairly common-sense level, like the ability to interact with familiar asymmetrical stimuli such as writing and remembered environments. These things would indeed look strange at first to Righty. But it's hard to believe that once Righty learned to adapt to these phenomena, and so became functionally the same as Lefty with respect to them, that would make him qualitatively left/right inverted with respect to his former self. Such abilities are very plausibly irrelevant to the orientation of our experiences-for example, people who have trouble remembering how to unlock their front door, surely can have normal left/right experiences. The case of memory of familiar environments is perhaps more controversial, and you might be tempted to conclude that Righty's inversion just consists in his finding these places to be different from how he remembers them. I think that would be a mistake too, although it would take us too far astray to argue the case in detail here. (A few suggestions are provided in the footnote below. $)^{13}$ But my main interest is with certain consequences of the Relationist Argument that follow whether or not this should be classified as a genuine case of inverted qualia, as that is normally understood.

Below we will see what happens if you think that absolute reflective facts exist and are relevant to what experiences we have. But first I want to point out one interesting and paradoxical-seeming consequence of the Relationist Argument.

\section{SYMMETRICAL OBSERVERS}

The conclusion of the Relationist Argument was that if we were to construct a molecule-for-molecule physical duplicate of Lefty at the time when his eyes are

13 The position would have to be that experiencing an environment as having one orientation rather than another consists of an input representation being compared with memory (the congruence relations being calculated, and so forth). Against this, it seems plausible that: (1) someone would have different experiences of incongruent stimuli even without stored information about his environment, for example, if he were a baby or an amnesiac; (2) the visual processing on which the basic spatial content of experience depends does not involve a 'top down' memory component of this kind, but is wholly 'bottom up'; and (3) if left/right determinate experience requires memory in this way, then your first experiences could not be left/right determinate- but then how could memories formed from them serve as a basis for the left/right determinacy of later experiences? 
closed, but arrange the matter in an exact mirror image of the way it is organized in his actual body, the resulting mirror twin would, on opening his eyes, have experiences that were reflectively inverted with respect to Lefty's. However, this result raises the following puzzle: what if Lefty happened to be constructed symmetrically? 14 In particular, what if his brain, the organ responsible for his experiences, was constructed symmetrically? On the face of it, there seems to be no reason why such a design could not be totally adequate for this organism to achieve everything we actually achieve. Let us suppose that we have such a symmetrical beast, and let us christen him 'Simple Simon' ('Simon' for short).

The problem now raised is that if we were to produce a mirror twin of Simon, he would be in all intrinsic respects physically the same as Simon. So, provided we are assuming that some reasonable kind of mental/physical supervenience thesis is true, we would expect Simon to have the same kinds of experiences in the same situations as his mirror twin. (In particular, if we consider Simon and mirror Simon gazing at a scene from the same perspective at $\mathrm{t} 2$, having had their eyes shut at $\mathrm{t} 1$, then, since they are intrinsically the same at $\mathrm{t} 1$, they'll still be intrinsically the same at $\mathrm{t}$ 2.) But if we were to assume that symmetrical Simon's experiences depend on the world in much the same way that ours do, then we are also led by the Relationist Argument to conclude that they would be different from his mirror twin's. So the assumptions of the Relationist Argument seem to lead to a contradiction.

To clarify, note that Simon is only assumed to be symmetrical immediately prior to being imprinted with experiences. Of course, once the river of experiences starts flowing we would probably expect asymmetries to appear in the brain, perhaps depending on how the brain is constructed. For example, you might expect an experience of an asymmetrical landscape to require an asymmetrical pattern of electronic activity in the brain. The point is that if Simon is initially symmetrical, then at the time of experience he will be an exact mirror duplicate of the way he would have been had the environment been arranged mirrorwise. The puzzle is that if we suppose that absolute reflective facts are irrelevant to determining experiences, then Simon would have the same experiences whether the environment was arranged one way or the other.

In terms of our 'toy model' for experience, the situation is like one where both sides of our imaginary subject's head are painted the same colour. If that were the case, then his forehead would end up in the same state (up to incongruence) whichever of two incongruent stimuli we showed him. So he would have qualitatively the same experiences of the two stimuli.

Clearly, the only thing that someone who wishes to endorse the Relationist Argument can do to avoid contradiction is to bite the bullet and say that Simon has the same experiences of incongruent environments. We could describe this consequence as 'geometrical symmetry entails experiential symmetry'. Someone

14 Again, there is a problem here with asymmetrical laws of nature, which I will discuss later. 
is experientially symmetrical 15 if her (veridical) experiences of incongruous environments are the same. Another way of putting this consequence is that asymmetrical experiences are necessarily underwritten by an initial asymmetry in the subject's brain. And I think that if this were true, that would be quite surprising.

Even if we can't imagine from the inside being experientially symmetric, it seems there is nothing obviously incoherent about the idea, even if it is quite puzzling. For example, Rory Madden described to me how when he was a child he was puzzled by the idea of colour blindness. If someone can't tell the difference between green and red, but their colour discriminations are otherwise normal, surely this means that their experiences of both red and green things must either be both like a normal person's red experiences, or both like a normal person's green experiences. And then of course the puzzle is: which is it? But, as he concluded, evidently the answer to this puzzle is that they are neitherthe colour-blind person's experiences are just less determinate than ours with respect to colour. And similarly with symmetric experiences—even though we can't imagine seeing an environment without seeing it as oriented reflectively one way or the other, it seems at least prima facie possible that someone else's experiences could just be less determinate than ours in this respect. (To approximate to what their experiences would be like, try imagining having two visual fields, one oriented one way, and one the other. Or, even better, try imagining having a patchwork of infinitely many visual fields arranged so they alternate spatially in orientation. ${ }^{16}$ (The key thing is to not make the mistake of thinking that symmetrical experiences would be like our experiences of symmetrical environments.))

So there is sufficient reason for not dismissing the idea of experiential symmetry offhand. However you might wonder whether it's really true that symmetrical Simon would have these experiences. There are two kinds of strategy for casting doubt on this. First, you might simply attack the specific argument we have for this claim, for example, by supposing that absolute reflective facts exist and are part of what determines our experiences. Second, you might argue that the conclusion is false on independent grounds, hence casting doubt on the original argument. I'll first discuss a worry of the latter kind.

As I mentioned briefly above, it seems offhand that a brain whose initial design (i.e., prior to experience) was symmetrical could potentially achieve anything that our brains can achieve. There is no obvious reason for supposing that this design would necessitate any limitation on the range of psychological features that the brain could support. It therefore seems bizarre to suppose that this brain could not produce left/right experiences anything like ours.

An important example of a psychological feature that Simon clearly could have, the existence of which jars with the view that his experiences would be

15 This bit of terminology was suggested to me by Ned Block.

16 Thanks to John Hawthorne for suggesting the infinite version of the 'multiple visual fields' idea. 
symmetrical, is the following: it seems like Simon could perfectly well intentionally move towards an object on his right, and it be true that had the object been on his left he would have moved instead to the left to reach the object. So Simon's perceptual system could be quite capable of sustaining the normal kinds of dependence between a bodily action and the location of the target of that action. But you might wonder whether that fact is consistent with the claim that the experience of the object would be the same whether the object was on the left or the right. ${ }^{17}$

You could respond to this as follows. First, it has to be admitted that there is after all at least one important psychological limitation that is imposed by symmetry. This is that an initially symmetrical person like Simon could not make any classificatory distinctions between things on the left and on the right. ${ }^{18}$ If you ask Simon to say which side of his visual field an object is in, left or right, then he could not tell you. Equally, if you presented him with a word containing only symmetrical letters like 'TIM' he could not tell you whether it said 'TIM' or 'MIT'. This is because we would expect the only physical difference between Simon and his behaviour with respect to incongruous stimuli to be itself just incongruence. ${ }^{19}$ But it seems like such classificatory differences would require more than just incongruence. It seems that in order to make such a classificatory distinction there would have to be a leftright asymmetry in the brain that was exploited to distinguish the two stimuli, in a way somewhat analogous to the way a lock picks out a key.

Does this show that Simon has symmetrical experiences? Probably not. On the face of it, experiencing a difference between left and right, and being able to classify that difference are two quite different things. For example, being able to say whether an object is on the left or right is a skill that is surprisingly difficult to acquire. But we would not take a lack of such classificatory skills in a child to be evidence that her experiences were not left/right determinate. So, if there is strong connection between the content of experience and the ability of the subject or the brain to classify information, that is something that is unobvious and must be argued for. (Perhaps one way of articulating the debate over whether experience could have non-conceptual contents would be in terms of this distinction between content and classification.)

Indeed, doubt about such a connection is cast by the point about Simon's actions. The fact that Simon would walk in the correct direction to reach an object whether it is on the left or right suggests that there can be evidence that supports the attribution of a left/right distinction to the subject's experience that does not involve him classifying that difference.

17 A closely related point is that there is a sense in which incongruous subjects are functionally different-their dispositions to move either to the left or right, for example, are different, so the 'output clauses' given in the functional characterization of their mental states would be different. This might be thought sufficient for a difference in their experiences. (Also, see discussion on p. 309-11).

18 I am grateful to Ned Block for pointing this out to me.

19 Again, modulo the effects of asymmetrical laws of nature, which I will discuss on p. 311-12. 
The relationist might respond to that point by saying that the two situations of the object being on the right or the left could only relevantly differ if there was some asymmetry in the subject's body prior to experience. For example, he will say that the difference between a subject being embedded in an environment as opposed to its incongruent counterpart, is not that the two environments are intrinsically different, rather that they differ in their relations to the subject's body. But these relations only differ if the subject is left/right asymmetrical prior to experience. Otherwise the two scenarios are really the same, and so there is no difference in behaviour that needs explaining. (This response seems to me sufficient to remove much of the force of this observation.)

A final point about Simon is that unlike a colour-blind subject, it is plausible that he might be able respond differentially to successive presentations of congruent or incongruent stimuli. ${ }^{20}$ For example, if you showed him 'MIT' followed by 'TIM', he could tell that the second stimulus was different, and he would say that his second experience was qualitatively different from his first. This is so, even though on the relationist view, had he been shown the opposite sequence of stimuli ('TIM' then 'MIT'), he would have had qualitatively the same sequence of experiences. Now it seems that we must say that the second experience is asymmetrical, otherwise we risk scepticism about whether our experiences are asymmetrical. ${ }^{21}$ But then it seems surprising to claim that the first experience was not also asymmetrical. Indeed, the problems seem to start even earlier. To what extent is it coherent to speak of the 'first experience'? (How long did it last?) And, even supposing that there is such a thing, how could it introduce the required asymmetry into Simon's brain? For, if we suppose (as I suggested in n. 13) that the basic spatial content of experience depends only on 'bottom up' processing, and not on memory, then one might expect the relevant part of the brain to 'reset' itself prior to each new train of experience. This view of the processing, combined with relationism, would result in the view that each new train of Simon's experience would be symmetrical, even though Simon would be able to discriminate between incongruent stimuli, and would think that his experiences of them were different. In light of this, the relationist may be forced into the view mentioned in $\mathrm{n} .13$, that the existence of the left/right content of experience depends on memory. ${ }^{22}$ This seems to be a fairly forceful consideration against the view.

I now move on to consideration of what happens if we reject the main premise of the Relationist Argument, and suppose that absolutism is true.

${ }^{20}$ Not every symmetrical being would necessarily have this capacity. But there seems to be nothing about symmetry to prevent you from having such a capacity.

${ }^{21}$ If Simon's subsequent experiences were not asymmetrical, he would still think that they were. But then how would we know that we were not in the same position as Simon?

${ }^{22}$ If this is right, then perhaps the relationist should reject my claim that adjusting to asymmetrical stimuli like writing and remembered environments would not invert Righty's experiences. This would weaken the sense in which Righty could be said to be non-manifestly inverted. 


\section{WHAT THE ABSOLUTIST MIGHT SAY}

The main premise of the Relationist Argument is that the experiences had in perfectly incongruent scenarios are qualitatively the same (at least with respect to left/right orientation). For example, it was claimed that the experiences had by Lefty in scenario (1), and the experiences being had by Righty in scenario (2), would feel the same. Now, as I have already remarked, an absolutist about the left/right orientation of space could accept this premise, and so could accept the conclusions of the Relationist Argument, provided they don't think that the intrinsic difference that they believe exists between incongruent counterpart scenarios is relevant to determining the reflective character of any experiences being had in those scenarios. On the other hand, they could also reject the main premise of the argument, by insisting that this intrinsic difference does make a relevant experiential difference.

If there is an intrinsic physical difference between scenario (1) and scenario (2), then global supervenience alone will not tell us anything about the relationship between the experiences in the two scenarios-supervenience is compatible with the experiences being completely different. However, it is reasonable to assume that both subjects are correctly perceiving the spatial layout of the objects in the same amount of detail (perhaps modulo the reflective orientation of the objects in space-we can leave open the possibility that one of the subjects is perceiving this incorrectly). Given this assumption, we can intelligibly compare the reflective orientation of their experiences - either it is the same or it is different. The main premise of the Relationist Argument is that it is the same, because the experiential facts do not depend in any way on absolute spatial facts (if there are any at all). The most plausible alternative ${ }^{23}$ to this relationist view would be to hold that in general the orientation of experiences being had in perfectly incongruent scenarios like (1) and (2) is different-that is, the experiences are opposite in orientation.

I will call this view $A$ bsolutism with a capital 'A'. (Note that to be consistent with global supervenience, this view requires that absolute reflective facts exist and that orientation of our experiences depends on them.)

We can illustrate the Absolutist view by considering again the case of incongruent counterpart brains. Would the owners of these brains have experiences that feel the same? Provided that the Absolutist thinks that the qualitative character of experience supervenes narrowly on the intrinsic state of the brain, the Absolutist's view is that the experiences had by the owners of these two brains are opposite in their reflective character, because the brains are identical except for the intrinsic reflective difference between them.

${ }^{23}$ Strictly speaking, you could also hold the view that in some pairs of cases like (1) and (2) the orientation is the same, and in some pairs of cases it is different. Most of my discussion of Absolutism will also apply to this view, which suffers further difficulties I will not discuss. 
But, even if he does not assume any narrow supervenience hypothesis, the Absolutist can still say, for example, that the experiences being had by Lefty in scenario (1) and Righty in scenario (2) are relevantly different because the two scenarios are perfectly incongruent, both with respect to the intrinsic set-up of the observers and their environments. And that is one way to block the Relationist Argument.

It is interesting to note that it would follow from Absolutism that Righty and Lefty would have the same experiences of actual stimuli, and so would not be reflectively inverted with respect to each other after all. For example, according to Absolutism, when Righty sees 'MIT' he is experientially inverted with respect to Lefty seeing 'TIM'; so if we show them both 'TIM', they will have the same experience of it.

I will shortly assess the plausibility of holding this Absolutist position. But let us first ask: where would an Absolutist stand on whether the Inversion Hypothesis is true? The answer depends on what kind of supervenience thesis about the qualitative character of experience he thinks is true. If he thinks that what it's like for the subject depends only on what's going on in the subject's brain (the 'narrow supervenience' view), then he should think that inversion is possible. If he thinks that a more external dependence holds, then he might reasonably think that inversion is not possible.

As I have just remarked, on the narrow supervenience view, the Absolutist is committed to the view that the owners of incongruent conscious brains will be having mirrorwise different experiences. And now it's not that hard to see how you could set things up so the states of two such brains would be caused by the same external stimuli under normal circumstances, so that inversion is possible.

For example, we could take Righty, and literally cross over all the neural wiring in his body leading from brain to sensory neurons at the surface. For example, we would reroute the neural connection that goes from left thumb-tip to brain so that it goes from right thumb-tip to brain, and so forth. We could also similarly rewire all the motor neurons, so that he could move around successfully. Once rewired this way and given a particular stimulus, what would happen in his brain during experience would be physically a mirror image of what would happen in Lefty's brain given the same stimulus. ${ }^{24}$ So if it is true that brainwise physical inversion (at the time of experience) entails experiential inversion, as we are now assuming, then 'doctored Righty' is experientially mirror inverted with respect to Lefty.

Suppose we thought instead that certain external facts are part of the minimal base of facts on which the qualitative character of experience supervenes, so that narrow supervenience is false. If the Inversion Hypothesis is true, then there could be inverted experiences caused by the same stimulus under normal conditions. But the stipulation of same stimulus and normal conditions might mean that the

24 Again, modulo asymmetrical physical laws, which I will discuss shortly. 
relevant external facts are the same, depending on which ones we are putting in the supervenience base. So the Absolutist externalist could argue that two subjects under these conditions must be having reflectively the same experiences. So the externalist Absolutist could argue that inversion is impossible.

If we are absolutists (small 'a'), then it is certainly plausible that such externalism is the correct view about the left/right content of experience. For example, you might think that if an experience of an object that presents it as 'phenomenally left' is also caused under normal circumstances by the object being actually on the left, then the experience represents it as on the left. (For the relationist, the story about content might be more complicated—unfortunately, I can't get into that here.) If something like that is right about the left/right content of experience, then there is one interesting brand of Absolutist externalism that can be viewed as saying that what it's like to see something as on the left is exhausted by the fact that it is being represented as on the left. That may sound plausible until we consider the possibility of left/right inversion. If inversion is possible, then content externalism could mean that inverted subjects' experiences have the same spatial contents, even though they are phenomenally different with respect to left/right presentation.

It's worth noting how much this kind of Absolutist externalist view of the qualitative character of spatial experience jars with the unreflective view most people would take. It would imply that it is possible for two subjects to be in the same brain state, and both be having veridical experiences, but nonetheless for the qualitative character of their experiences be different. That would be the situation, for example, if we compared Righty viewing one environment with 'doctored Righty' (as defined three paragraphs above) viewing the incongruous counterpart of that environment. Since the environments are incongruous and we can assume that both subjects are having veridical experiences with absolute left/right content, their experiences must differ in content, and hence, according to this view, also in qualitative character. ${ }^{25}$ But their brain states are identical.

I expect some people will take this view, but its metaphysical oddness should be acknowledged. The question of what the qualitative character of experience supervenes on is surely at least partly an empirical matter; but I take it that our common-sense empirical view is that sameness of the most proximate stimulus can be sufficient for sameness of qualitative character, so that the more distal facts could not be part of the minimal supervenience base. It would be a remarkable discovery if that turned out to be false.

The externalist view is sometimes motivated by the thought that experiences are relational states of affairs, and not simply monadic properties of subjects, where the relata are subjects and local states of affairs like a layout of words on a page being read, or an apartment being viewed. Since the same relation cannot

${ }^{25} \mathrm{I}$ 'm assuming here that this theorist is allowing that there can be a qualitative difference between seeing something on the left and seeing it on the right. That might be left open by my characterization of the view above. 
hold both in a case of veridical experience and a case of hallucination, these experiences must be different, the thought continues, even if the intrinsic brain states involved are the same. But to conclude from this that these experiences would be qualitatively different seems to me to be a non-sequitur-there is evidently no reason why experiences that involve relations to different states of affairs should not feel the same to the subjects having them. Equally, you might even think that being experientially related to the same state of affairs could feel different to different subjects. So it is evidently quite consistent to hold a narrow view of the supervenience of qualitative character along with a relational view of experience in this sense. ${ }^{26}$

So much for Absolutism and the Inversion Hypothesis. We now need to ask: could Absolutism really be true?

The idea that these absolute spatial properties could make any difference to experience might plausibly be thought as absurd as the idea that if the whole universe suddenly spun around 180 degrees in absolute space that could determine some change in our experience. This intuition can be brought out especially strongly if we consider the fact that reflection is not a mathematical operation of a substantially different kind from rotation or linear translation. In fact, it can be shown that reflection is just an instance of a linear translation and rotation, albeit through a higher dimension. ${ }^{27}$ Hence it would not be inaccurate to say that the difference between a subject gazing at my apartment and a mirrorimage copy of the subject gazing at the mirror apartment is merely a difference in the orientation of the two environments (the subject included as part of the environment). But how could a mere difference in the orientation of the subject and his environment constitutively determine a difference in experience, even if these orientations could be thought of in absolutist terms? Again, we would strongly resist this idea if the orientation in question was, for example, just the location and direction of the subject and his environment in $3 \mathrm{D}$ space. ${ }^{28}$

\footnotetext{
26 A different 'relational' position about experience (see, for example, Johnston 2004) is one that insists that the qualitative character of the experience is exhausted by and uniquely correlated with the structured array of properties and relations one is related to in having the experience (rather than the more concrete particulars and states of affairs). But that is not necessarily incompatible with narrow supervenience, especially if one thinks that you can be acquainted with such an array in having an hallucination. Is this position correct? It would be close to being the right view if it could be argued that whenever one's experience has a certain sensory quality, such as that involved in seeing a red object, one is ipso facto experientially related to a correlative secondary property such as redness. But if one thinks of a case like left/right inversion, it is clear that sometimes one can motivate the idea that experience has some qualitative aspect, without it being in any way natural to think of tokenings of that quality as involving the subject being experientially related to some secondary property.

27 See Van Cleve (1991) for discussion of this point, which was originally made by the mathematician Moebius_-see Moebius (1991).

${ }_{28}$ Perhaps if absolutism is true then a change in orientation might be causally sufficient for a change in experience, but that is obviously not to the point. The present claim would have to be that there would be a change in experience, even if all the other physical facts were held constant (even if that is nomologically impossible).
} 
I would suggest that if we think that reflection is a significantly different kind of operation from these other spatial operations like rotation, that is because it appears to us that the difference we experience between incongruent objects is of a different kind from that we experience between, say, mutually rotated qualitatively identical objects. But when we just look formally at these operations, the intuition this appearance suggests is not supported - and it should be clear by now that we do not need to bring in some magic difference in the operations to explain these experiential facts. It could be that the right way to think about it is that seeing a difference between incongruent objects is a bit like seeing exactly similar congruent objects, but from different sides, i.e., in situations where our relations to the objects differ, not the objects themselves.

Another very different objection to the Absolutist view is that it is inconsistent with certain ways of being a functionalist about experience. Absolute spatial facts are facts that on many views could differ across worlds without any relevant difference in the causal profiles of those worlds. ${ }^{29}$ So, for example, you might think that if absolutism (small 'a') is true, then there is a world just like our world except that it is arranged in a mirror image. The Absolutist is committed to the view that the experiences being had by your counterpart in that world are quite different from your experiences, even though all the functional facts in these two worlds are the same. ${ }^{30}$

Another way of bringing out this point is to consider a thought experiment by Ned Block, as updated by Arnold Zuboff. ${ }^{31}$ It seems that in principle we could remove each of your brain cells one by one and fit each removed cell and the adjacent cells with minute radio transmitters that were capable of ensuring that the cells continued to fire exactly as and when they would if still embedded in the ordinary way. It is very hard to see how this could make any difference to the experiences had by the subject. ${ }^{32}$ This suggests that we could in principle put the cells of your brain in any spatial arrangement that we fancied without this affecting how your experiences ensued, the relevant functional organization being preserved by the operation.

If this is right, then the spatial arrangement of the brain seems irrelevant to the determination of experience after all. For example, if we were to take Lefty and doctored Righty, and explode their brains à la Block/Zuboff, we would end up with the same system of transmitters connected to the body in the same way. So, if the intuition generated by the Block/Zuboff experiment is correct, then the

29 That would not be allowed for on a 'causal essentialist' view like Shoemaker's (1980), however.

30 Actually not all functionalists need say this-if we consider 'local' functional characterizations of you and your counterpart, with input and output clauses that are allowed to mention absolute spatial properties, then we could get different characterizations for you both. We only get in trouble if we 'Ramsify' out over the input and output clauses also.

31 See Block (1980) and Zuboff (1981).

32 Admittedly, Block's original thought experiment was designed to provoke the opposite intuition. 
absolute spatial difference between Lefty's and doctored Righty's brains, whilst undergoing counterpart experiences, could not make any difference to those experiences. So Absolutism is false.

You could try making a similar point about the supposed requirement of asymmetry that was established from the premises of the Relationist Argument. You might think you could apply the Block/Zuboff technique to any normal subject to render him temporarily symmetrical, and his subsequent experiences would still differentiate between incongruent stimuli. (I am unsure about whether this objection to a relationist position could be made to work, but it's interesting to contemplate. ${ }^{33}$ )

The Absolutist might respond as follows. Suppose we take a complete description of our universe, and then 'Ramsify' over it, replacing all the property and relation names with variables, except those that refer to the causal relation. Is it really plausible that if we bleach out everything in this way, including even terms for spatial properties and relations, we'll end up with a description that completely determines which experiences are occurring? We wouldn't think that if we 'Ramsifyed out', even over the causal relation as well, so that the description of the universe was completely formal. So what's so special about the causal relation? ${ }^{34}$ For example, we can imagine a computer simulation of the actual world that satisfies the same Ramsey description, but does not literally contain the same distribution of spatial properties. And it is at least not obvious that running such a simulation would produce real experiences. So, viewed from an abstract standpoint, it is not particularly compelling that spatial properties could not be relevant to the determination of experience.

Similarly, a Block/Zuboff exploded brain shares a certain Ramsey characterization with a normal brain-they are in a certain way functionally the same, even though they can massively differ in their spatial properties. Unlike in the case of functionally equivalent universes, the functional equivalence here is only at a relatively macroscopic level—-the brains are not functionally the same at the level of fundamental particles, for example. And the brain is not a causally closed system, so the relevant functional description may be in terms of substantially specified sensory inputs and behavioural outputs; and there are other related differences like the fact that only a normal brain can be stroked or stamped on. But these differences can hardly make all the difference. Unless we

33 Suppose, for example, that having an experience of a certain stimulus involves a sequence of synchronous neuron firings, each synchronous firing involving 10,000 neurons. Suppose that had the experience been of an incongruent stimulus, there would have been a completely different sequence of synchronous firings, each synchronous firing instead involving 9,800 neurons. Then there will be no way to set things up using the Block/Zuboff technique so that the pattern of neural firings would be the same up to incongruence whichever stimulus is presented. However, in order for the point to go through, only one possible case is required, so this consideration is far from decisive. It is unclear, however, how one would establish the existence of such a case. (One suggestion, made to me by Tamar Szabó Gendler, would be to try making replacements in a way that was not one-one.)

${ }^{34}$ John Hawthorne (2001) makes this kind of point. 
are externalists who think that the only spatial facts that matter to experience are those external to the brain, then if we think that it is dubious whether the spatial properties really are irrelevant in the universe case, we should probably think the same about this more localized version.

\section{ASYMMETRICAL LAWS OF NATURE}

So much for considerations for and against the view that absolute reflective properties are relevant to experience. One final issue that is opened up by supposing absolutism (small 'a') is that natural laws might be sensitive to the left/ right distinction. ${ }^{35}$ So, for example, it might be a law that all fundamental particles are shaped like left gloves. ${ }^{36}$ This would be relevant to our discussion if it meant that making perfect mirror-image copies of objects is physically impossible, since both the Relationist Argument and the Absolutist considerations for intra-world inversion relied on the idea that such things are possible (remember that an absolutist can run the Relationist Argument.) Similarly, it might mean that making a perfectly symmetrical object like Simon is physically impossible. Finally, it would also be relevant if it meant that systems that start off as incongruent counterparts do not necessarily evolve in the same way. For example, I assumed earlier that if you show symmetrical Simon a certain stimulus, then the state he would go into would be the incongruent counterpart of the state that he would have gone into had he been shown an incongruent counterpart stimulus. If the laws are asymmetrical, this counterfactual claim might not be correct.

These impossibilities are worrying because we might be forced to locate these thought experiments in worlds with different natural laws to ours, worlds where perhaps we should not even be confident that left/right experiences are possible at all. One response would be to claim that only relatively macroscopic mirroring is required for the thought experiments to run, because experiential facts depend only on properties at some relatively coarse level of grain, such as

35 It is very plausible that there could only be such laws if absolutism was true. Otherwise the only way the asymmetry could be created by a law of nature would be if a canonical formulation of the law made explicit reference to a sample asymmetrical object, and then fixed the asymmetry universally using the congruence relation. It would surely be very surprising if a law looked like that, since we do not think that fundamental laws mention particular objects.

36 It turns out that the empirical evidence suggests, somewhat surprisingly, that the universe is indeed ruled asymmetrically in this way. So there is empirical evidence that absolutism is true. Changing the reflective parity of an atom would change the charge of the particles that constitute it, transubstantiating it from matter into anti-matter. And the laws governing matter and anti-matter are rather different (although I am told that an invariance does exist if we consider the trio of variables: charge, reflective parity, and time. So one could still hold a more complex form of relationism, consistently with these observations). In particular, given laws governing the interaction of matter and anti-matter, if you produced a mirror-image copy of an object it would disappear in a flash of energy before it had the chance to have any experiences. 
neural properties. These coarser properties could presumably be laid out in a mirror image even if the fundamental properties couldn't be.

But the problem with this is that what experience supervenes on is an empirical matter, and so it's not clear why we should trust these intuitions, given that they do not appear to be backed up by any empirical evidence. ${ }^{37}$ We would do better to just insist on running our arguments in worlds where the laws of nature are not of this kind. If an opponent were to press the complaint that we have no reason to suppose left/right experience is possible in these symmetrically ruled worlds, we can throw down the following challenge. If these experiences are not possible in such worlds, then that must be because there is some physical property required for left/right experience that can only be instantiated in worlds that are ruled asymmetrically. But at first blush that is just an extreme example of an 'initial asymmetry' requirement. ${ }^{38}$ So it is hard to see how a position about left/right experience distinct from those we have already considered could be generated from bringing these laws into play.

Certainly more discussion is needed of this issue. It's not obvious in what sense having such an asymmetrically profiled property would have to make you spatially asymmetrical, so the position may be slightly different from the one generated by the Relationist Argument. There may also be an interesting way of denying Inversion by taking such a line-it could be claimed that the property required for having experiences with one phenomenal orientation could not be instantiated in the same universe as the property required for having experiences with the other orientation, since the instantiation of these properties requires different causal laws. (Without further argument, such a position strikes me as rather implausible, but it does represent an epistemically possible way things might be.)

\section{TRANSCENDENTAL INFERENCES}

If I have been successful, the argument so far should have convinced you of the following: that however we look at it, there are some interesting connections between the geometrical properties of the world, and the experiential properties that are instantiated in the world. And these connections seem to have been established on an a priori basis given only some very slim assumptions about the nature of experience.

We can summarize these findings as follows: if absolute reflective properties do not affect the qualitative character of experience, then the experiences being

37 It's worth noting here that authors such as Penrose have claimed that facts at a deep level could be relevant to experience, so clearly not everyone shares the intuition that only relatively macroscopic features of the world are relevant to experience.

38 Notice that it wouldn't be a version of Absolutism, because there can be absolute reflective facts in symmetrically ruled worlds. 
had in perfectly incongruent scenarios are qualitatively the same. It follows from this that a subject who started life as a mirror image of you, but who received the same stimuli as you, would subsequently be experientially inverted with respect to you; and this shows that experiential asymmetry requires a prior physical asymmetry in the subject. On the other hand, if conversely Absolutism is true, then the experiences being had in perfectly incongruent scenarios are reflectively opposite, and so the qualitative character of experience depends in a certain way on the absolute spatial facts. Furthermore, the truth of the Inversion Hypothesis is entailed by Absolutism, provided narrow supervenience obtains. If it does not obtain, it is left open by Absolutism whether the Inversion Hypothesis is true. In sum, whichever way we look, there seem to be tight connections between the spatial properties of the subject (or on an externalist view, the subject and/or his environment) and the associated experiential properties.

It's certainly surprising that we could have discovered all this a priori from a few extremely uncontroversial assumptions about experience. Could I really work out, just sitting here, that my brain is asymmetrical? Perhaps if you take an externalist line such as the following - that the spatial character of the experience is inherited from the spatial properties of the things experienced-then you shouldn't find it surprising how easily a connection between space and experience is established. (This might be evidence in favour of the externalist view.) But even the externalist is faced with the possibility that we have an armchair argument to the conclusion that either Absolutism is true, or the surprising consequences of the Relationist Argument follow. And that disjunctive conclusion alone is surprising as a candidate for armchair accessibility.

To decide whether we really have acquired such knowledge from our armchairs, and if so what kind of knowledge it is (is it empirically defeatable, for example?), would require a long discussion about epistemology which is beyond the scope of this chapter. But, at first blush, the argument seems to proceed from known premises using moves that are warrant transferring. So the burden of proof appears to be on the person who thinks that the argument is not knowledge conferring.

\section{CLIMAX AND CONCLUSION}

I have now looked at the competing ways of drawing out the connections between the spatial properties of brains and the left/right character of experiences, and suggested some considerations for and against different ways of thinking about the problem.

Now for the climax of the paper. One of the following pictures of left-right experience must be correct, even though each is in its own way quite bizarre. We have, in short, what Aristotle called an 'aporia' - a choice of views, none of which seem true, accompanied by knowledge that one of them is. So, by way of 
a summary, here are the main contenders ((III), (IV) and (V) simply result from denying assumptions made in the paper until this point):

\section{The Five Options}

(I) To say that if there are non-relational reflective properties, then they are irrelevant in determining experiences, and hence to accept that a physically symmetrical individual like Simon would be experientially symmetrical.

(II) To be an absolutist about the reflective orientation of the world, and claim that this orientation would be sufficient to determine a difference in the subject's experiences in our various cases.

(III) To deny that there is ever any qualitative difference between a veridical experience of an environment and its mirror image by the same subject.

(IV) To deny the supervenience of experiential properties on physical properties.

The fifth option is to endorse what Shoemaker calls the 'Frege-Schlick' hypothesis.

(V) To deny that there are any inter-personal, inter-world, or inter-temporal facts about the sameness and difference of the qualitative character of experiences.

Of the five, options (III) and (IV) are the least plausible. Option (III) requires the barefaced denial of an apparently incontestable feature of experience. Andunless it is tantamount to commitment to a free-floating realm of experience largely independent of the physical world-it is unclear that (IV) really helps, for, even if the physical to mental dependencies are merely nomological, it appears we could run the same arguments considering the causal basis for experience instead of the supervenience base.

What about $(\mathrm{V})$, the Frege-Schlick view? The view is motivated by the thought that what makes particular experiences the same or different is simply how a subject would classify them if confronted with them; this immediately rules out inter-subjective comparisons of experiences. ${ }^{39}$ But, to me, simple examples from everyday life seem sufficient to establish that such comparisons are possible, so the view seems like a last resort. Others disagree, however ${ }^{40}$ so perhaps the view warrants further investigation.

This leaves us with (I) and (II). I argued that (I) entails that inter-subjective reflective inversion is possible, although we were left with some doubts about that. ${ }^{41}$ Option (II) less controversially entails this, provided we assume that the qualitative character of experience supervenes on the intrinsic set-up of the

\footnotetext{
39 Although see Shoemaker (1982) for an attempt to hold on to this thought without accepting the Frege-Schlick view.

40 For a recent dissenter, see Stalnaker (1998).

${ }^{41}$ See in particular n. 22, and the surrounding discussion.
} 
observer's brain. (If we reject that assumption, we get the 'externalist Absolutist' view that I have mentioned, which is compatible with denying the possibility of inversion.)

I think that these views are the only serious contenders. Each brings surprising consequences about the physical basis of experience, yet one of them must be true. Which way should we go?

\section{REFERENCES}

Block, Ned (1980), 'Troubles with Functionalism', in Ned Block (ed.), Readings in Philosophy of Psychology, Volume 1 (Cambridge, Mass.: Harvard University Press): 268-305.

Davies, Martin (1992), 'Auntie's Own Argument for the Language of Thought', in J. Ezquerro and J. M. Larrazabal (eds), Cognition, Semantics and Philosophy: Proceedings of the First International Colloquium on Cognitive Science (Dordrecht: Kluwer Academic Publishers): 235-71.

Hawthorne, John (2001), 'Causal Structuralism', Philosophical Perspectives 15: Metaphysics (Oxford: Blackwell): 361-79.

Hurley, Susan (1998), Consciousness in Action (Cambridge, Mass.: Harvard University Press).

Johnston, Mark (2004), 'The Obscure Object of Hallucination', Philosophical Studies 120: 113-83.

Kant, Immanuel (1991), 'On the First Ground of the Distinction of Regions in Space', in James Van Cleve and Robert E. Fredericks (eds), The Philosophy of Right and Left: Incongruent Counterparts and the Nature of Space (Boston: Kluwer Academic Publishers): 27-35.

Moebius, August Ferdinand (1991), 'On Higher Space', in James Van Cleve and Robert E. Fredericks (eds), The Philosophy of Right and Left: Incongruent Counterparts and the Nature of Space (Boston: Kluwer Academic Publishers): 39-43.

Shoemaker, Sydney (1980), 'Causality and Properties', in Peter van Inwagen (ed.), Time and Cause (Dordrecht: D. Reidel): 228-54.

Shoemaker, Sydney (1982), 'The Inverted Spectrum', Journal of Philosophy 79: 357-81.

Stalnaker, Robert (1998), 'Comparing Qualia Across Persons', The Philosophy of Sydney Shoemaker, Philosophical Topics 26 (Denver, Colorado : Philosophical Topics Inc.): 385-404.

Van Cleve, James (1991), 'Left, Right, and Higher Dimensions', in James Van Cleve and Robert E. Frederick (eds), The Philosophy of Right and Left: Incongruent Counterparts and the Nature of Space (Boston: Kluwer Academic Publishers): 203-35.

Zuboff, Arnold (1981), 'The Story of a Brain', in Douglas R. Hofstadter and Daniel C.

Dennett (eds), The Mind's I: Fantasies and Reflections on Self and Soul (New York: Basic Books): 202-13. 